\title{
ON SIMPLE INJECTIVE RINGS
}

\author{
SIGURD ELLIGER
}

ABSTRACT. It is proved that a simple ring which is injective on both sides must be artinian. This answers a question asked by C. Faith in the negative.

In this note we will prove the following

THEOREM. A ring is the full ring of $(n, n)$-matrices over a field iff the following three conditions hold simultaneously:

(1) $R$ is simple,

(2) ${ }_{R} R$ is injective,

(3) $R_{R}$ is injective.

The proof depends on a result of Utumi [5]: Under conditions (2) and (3) of the theorem $x y=1$ implies $y x=1$. (The converse of this theorem holds if $R$ is a regular ring, cf. [4].) First we recall a

LEMMA. Let $R$ be a regular simple ring without primitive idempotents $\neq 0$. Then there are infinitely many pairwise orthogonal idempotents $e_{i}$ with $e_{i} R \simeq e_{k} R$.

For a proof see [2] or [3].

Proposition. Let ${ }_{R} R$ be injective, the left singular ideal $Z\left({ }_{A} A\right)=0$, $\left\{e_{i}, i \in N\right\}$ pairwise orthogonal idempotents with $\operatorname{Re}_{i} \simeq R e_{k}$. Then there exist $a, b \in R$ with $a b=1, b a \neq 1$.

PRoof. Define $R$-homomorphisms $f, g: \bigoplus_{i=1}^{\infty} R e_{i} \rightarrow \bigoplus_{i=1}^{\infty} R e_{i}$

$$
\begin{aligned}
f: & e_{2 n} \rightarrow e_{n}, \quad g: e_{n} \rightarrow e_{2 n}, \quad n=1,2, \cdots . \\
& e_{2 n+1} \rightarrow 0,
\end{aligned}
$$

It is clear that $e_{n}(g f)=e_{n}$ for all $n$, but $e_{2 n+1}(f g)=0$. From the lemma of Zorn $\left\{R e_{i}, i \in N\right\}$ may be imbedded in a maximal set $\left\{R e_{k}, k \in K\right\}$ of independent left ideals. Then $\bigoplus_{k \in K} R e_{k} \subset_{R} R$ essential.

Received by the editors July 6, 1972 and, in revised form, November 2, 1972.

AMS (MOS) subject classifications (1970). Primary 16A52, 16A40; Secondary 16A32.

(c) American Mathematical Society 1973 
The definition $e_{k} f=e_{k} g=e_{k}$ for $k \in K, k \notin N$ extends $f, g$ to homomorphisms $\bigoplus_{k \in K} R e_{k} \rightarrow \bigoplus_{k \in K} R e_{k} .{ }_{R} R$ injective and $Z\left({ }_{R} R\right)=0$ implies: $f, g$ may be extended in one and only one way to a map $R \rightarrow_{R} R$, that is $f, g$ are right multiplications by elements $a, b \in R$.

ProOf OF THE TheOREM. It is enough to prove that the conditions are sufficient. From the theory of quotient rings it follows that $R$ is a regular ring. Assume that $R$ has no primitive idempotents $\neq 0$. Then from the lemma and the proposition there must exist $a, b$ with $a b=1, b a \neq 1$. This contradicts the above result of Utumi. Thus $R$ has primitive idempotents $\neq 0$ and $R$ is artinian because $R$ equals its socle.

REMARK. The theorem answers a question of $C$. Faith in the negative. $[1$, p. 130, Problem 16.] As the referee informs me the same question was answered by E. Roos in a comment communicated to the referee: A simple right injective ring need not be left injective. Proof. Let $R$ be any integral domain, not a right Ore domain, and let $S$ be its maximal right quotient ring. Then, $S$ is known to be a simple ring which is regular and right injective. Moreover, by Utumi's theorem, $S$ cannot be left injective, since otherwise $x y=1 \Rightarrow y x=1$. Now, any $y \neq 0$ in $R$ has a left inverse $x$ in $S$, so this would imply that every nonzero element of $R$ is a unit of $S$. This is impossible, since given $s \neq 0$ in $S$, there exists $a \neq 0$ in $R$ such that $b=s a \neq 0$ in $R$, and then $s=b a^{-1}$. This would imply $R$ is a right Ore domain with right quotient field $S$, contrary to the assumption.

\section{REFERENCES}

1. C. Faith, Lectures on injective modules and quotient rings, Lecture Notes in Math., no. 49, Springer, New York, 1967.

2. N. Jacobson, Some remarks on one-sided inverses, Proc. Amer. Math. Soc. 1 (1950), 352-355. MR 12, 75.

3. I. Kaplansky, Topological representation of algebras. II, Trans. Amer. Math. Soc. 68 (1950), 62-75. MR 11, 317.

4. J.-E. Roos, Sur l'anneau maximal de fractions des $A W^{*}$-algèbres et des anneaux de Baer, C. R. Acad. Sci. Paris Sér. A-B 266 (1968), A120-A123. MR 39 \#6093.

5. Y. Utumi, On continuous rings and self injective rings, Trans. Amer. Math. Soc. 118 (1965), 158-173. MR 30 \#4793.

Institut für Mathematik, 463 Bochum Buscheystrasse, Postfach 2148, Federal RePUblic OF GeRManY 